\title{
One Step Chemical Synthesis of Carbon Dot Based Smart Fluorescent Security Ink
}

\author{
Jyoti Sharma \\ Institute of Research and Development, Gujarat Forensic \\ Sciences University, Gandhinagar -382007, India
}

\begin{abstract}
In the recent world, there are number of problems related to counterfeit of the different products. There are number of products, which can be easily duplicated by simple processes and the product is accurate and precise. Even the counterfeit of currency is also a main problem in world's economic. There are number of sectors, which can be effected due to this reason like duplication of electronic products, duplication of food products, duplication of automobile products, duplication of currency, etc. So there are number of anti-counterfeiting technologies available in the market to prevent counterfeit problems. In this paper, it explains to prevent this type of problems with the help of carbon dot based gel formulation, which can be used in different ways. This paper describes the synthesis process and characterization analysis of carbon dot based smart fluorescent security ink to prevent the counterfeit problems. UV-Visspectrophotometer was utilized to differentiate optical properties and fluoroscpectometer was exploited to differentiate fluorescent properties of carbon dots and carbon dot based fluorescent ink. AFM and FTIR were used to analysis the structural properties of carbon dots and carbon dot based fluorescent ink.
\end{abstract}

Keywords:- Carbon Dots, Fluorescent Ink, Security Ink, Anti-Counterfeiting.

\section{INTRODUCTION}

Carbon dots (CDs) have emerged as most precious gifts in nanotechnology because of their magical properties and applications. CDs are typically carbon nanoparticles, most of them with average diameter less than $10 \mathrm{~nm}$ [1],[2]. These materials are derived from organic compounds and are stable in aqueous media which is extremely significant in terms of biological points of view [3]. Surface engineering plays a significant role for CDs in diversified applications like explosive detection, chemical sensing, food safety, bioimaging, drug delivery, energy conversion, and photocatalysis. Photophysical and chemical properties of CDs vary dramatically by tuning their shapes and sizes and also by doping heteroatoms such as oxygen, nitrogen, phosphorus, sulfur, and boron [4]. Moreover, photostability, high quantum yield, biocompatibility, low toxicity, water solubility, good conductivity, and environmental friendliness of $\mathrm{CDs}$ receive additional advantages over other well-recognized quantum dots (QDs) like grapheme quantum dots (GQDs), metal oxides ( $\mathrm{ZnO}$, TiO2), and inorganic QDs (ZnO-PbS, CdSe, CuInS/ZnS, and $\mathrm{CuInS} / \mathrm{ZnS}$ ) [5]. In fact, noncarbon QDs are not much

\author{
Pranav Y. Dave \\ Institute of Research and Development, Gujarat Forensic \\ Sciences University, Gandhinagar -382007, India
}

graceful in their field of applications compare to CDs, because of their serious health and environmental issues. CDs can be synthesized from both natural and synthetic organic precursors. Synthetic methodologies that are very frequently used in this concern are microwave irradiation, hydrothermal treatments, ultrasonic irradiation, laser ablation, electrochemical, arc discharge, and pyrolysis [6],[7],[8].

\section{EXPERIMENTAL DETAILS}

\section{$>$ Materials and Method}

All the materials and solvents were purchased from commercial sources (Sisco research laboratories Pvt. Ltd., India) and used as received unless stated otherwise. Citric acid (anhydrous, extrapure AR, 99.5\%) and Ethanolamine (extrapure AR, 99\%) were obtained from SRL company and used without further purification process. Milli-Q water and spectroscopic grade solvents were used for all measurements. Fourier-transform infrared (FTIR) spectra were measured on a JASCO FT-4600 spectrophotometer by putting directly the sample solution into it. Solution phase optical absorption spectra were recorded on a JASCO V670 spectrophotometer by using a quartz cell with optical path length of $1 \mathrm{~cm}$ after diluting the sample in Milli-Q water. Steady state photoluminescence (PL) spectra were recorded using a JASCO FP-6500 fluorophotometer by using a quartz cell with optical path length of $1 \mathrm{~cm}$. All solution phase PL measurements were carried out at RT (room temperature) and without removing dissolved $\mathrm{O}_{2}$. Atomic force microscopy (Nanosurf C300) was used to find the particle size after diluting the sample into Milli-Q water and dried it on MICA sheet at room temperature. The gel was characterised by Brookfield viscometer after diluting the sample in 4:6 ratio with Milli-Q water.

\section{$>$ Synthesis Process of Carbon Dots}

The synthesis was followed by procedure reported by Gregory Ethan LeCroy et al.(2014) [5]. In brief, the one step hydrothermal process was used to synthesis crystalline carbon dots. 500mg of Citric acid (CA) was dissolved in $45 \mathrm{~mL}$ of Milli-Q water along with $1 \mathrm{~mL}$ of ethanolamine. The solution was mixed for few minutes on the stirrer and then it was transferred into the Teflon coated $90 \mathrm{~mL}$ of white chamber, which was a part of small autoclave system. After that the autoclave was heated along with the solution in it in muffle furnace at $175^{\circ} \mathrm{C}$ for $4-5$ hours. The solution was cooled and characterised by FT-IR and AFM for structural property. The fluorescence property was characterised by UV-Vis-spectrometer and fluorophotospectrometer. 
ISSN No:-2456-2165

Synthesis Process of Carbon Dot based Gel Formulation

The gel formulation was synthesised by literature reported by Apostolos Koutsioukis et al.(2019) [9]. Previously, the crystalline carbon dots were synthesised, which were mixed with commercially available water base varnish NAB polyurethane in ratio of 1:9. The process went overnight for better mixing of the sample and solvent. The commercial varnish contains a mixture of styrene-acrylicemulsion, acrylic resins, and emulsion of polyethylene wax.

\section{RESULT AND DISCUSSION}

\section{Characterization study of CDs}

For analysing the structural property of carbon dots, the XRD instrument was not useful because of the hydrophilic property of carbon dots. So the structural property was analysed by the FT-IR instrument. FT-IR instrument can detect the functional group of the material, so different materials can be detectable by the FT-IR graph. Figure.1 shows the FT-IR graph, which concluded the different carbon, oxygen and hydrogen bonds.

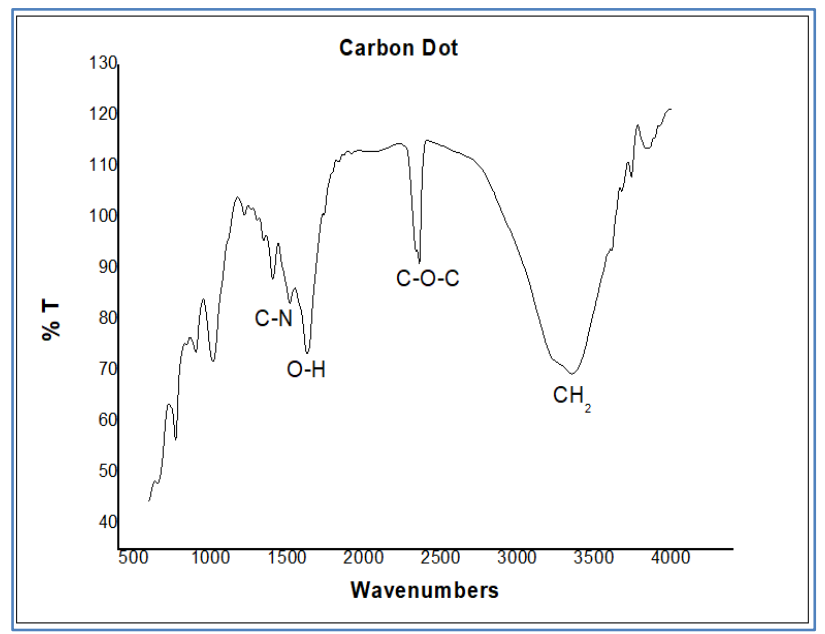

Fig 1:- FT-IR spectra of Carbon Dots

Here the absorption band $3450 \mathrm{~cm}^{-1}$ shows the presence of Methylene group, which correspond to the saturated hydrocarbon from amorphous carbonic framework in the CDs. And $3500-3100 \mathrm{~cm}^{-1}$ concludes the $\mathrm{O}-\mathrm{H}$ and N-H stretching vibrational peaks. Due to little bit vibration of the $\mathrm{O}-\mathrm{H}$ group, small peak can be detected on $3441 \mathrm{~cm}^{-1}$. The peaks at around bands $1600 \mathrm{~cm}^{-1}$ and $2400 \mathrm{~cm}^{-1}$ conclude the carboxylic group. Here another stretching vibration peaks found nearest to the $1500 \mathrm{~cm}^{-1}$ with $\mathrm{O}-\mathrm{H}$ and $\mathrm{C}-\mathrm{N}$ group. A strong absorption peak nearest to $2450 \mathrm{~cm}^{-1}$ is assigned to $\mathrm{C}-\mathrm{O}$ stretching vibration peak, revealing the existence of abundant $\mathrm{C}-\mathrm{O}$ or $\mathrm{C}-\mathrm{OH}$ groups. Further, the fluorescence property of CDs was analysed by the UV-Vis-spectrometer and Fluorophotospectrometer. Figure. 2 shows the UV-Vis spectrometer spectra of CDs. The graph shows some oscillator peaks between $300 \mathrm{~nm}$ to $500 \mathrm{~nm}$ wavelength.

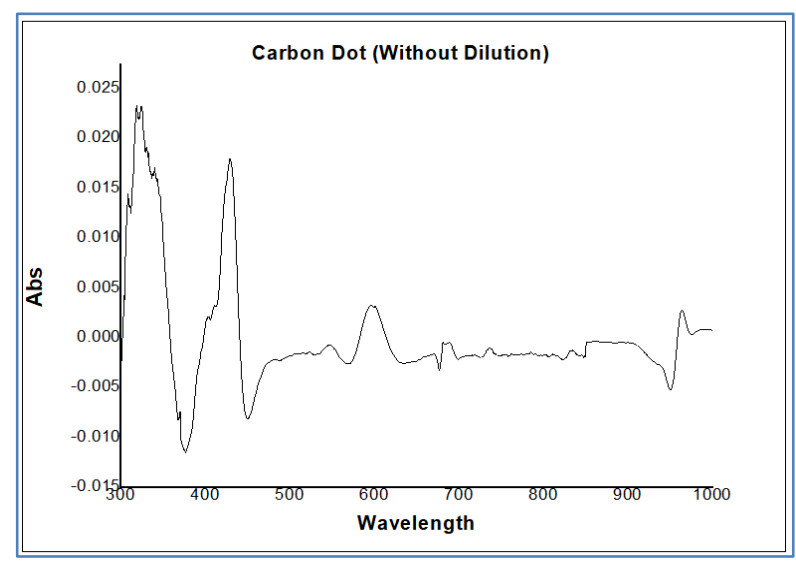

Fig 2:- UV-Vis spectra of Carbon Dots

The graph also indicates little bit dissociation energy after $650 \mathrm{~nm}$ wavelength. It has maximum intensity around $350 \mathrm{~nm}$ to $400 \mathrm{~nm}$ wavelength. The blue fluorescence absorbs at its highest intensity at $380 \mathrm{~nm}$ wavelength. But it is also depend on the parameters of the spectroscopy. Similarly, figure. 3 shows the fluorescence spectra of the CDs.

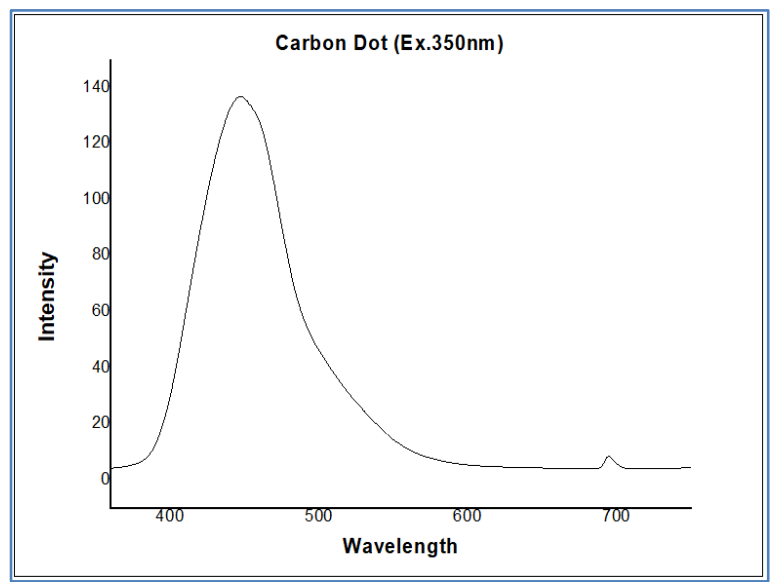

Fig 3:- Fluorescence spectra of Carbon Dots

Carbon Dots have their own excitation peak at 459nm wavelength. After putting the excitation wavelength $350 \mathrm{~nm}$ in spectroscopy parameter, the typical and diverse fluorescence absorption shows at the $459 \mathrm{~nm}$ wavelength. In contrast, the absorption peak of CDs in water is at $350 \mathrm{~nm}$ and the luminescence spectra between $400 \mathrm{~nm}$ to $500 \mathrm{~nm}$ show a maximum at $459 \mathrm{~nm}$ with strongest intense peak. The absolute QY of CDs in water is between $10-17 \%$ higher than of the pure CDs. When further increasing the absorption wavelength of CDs in water, the luminescence peak will become broader and shift to its original position. The CDs particle size is analysed by the AFM instrument. Figure. 4 shows the AFM image of CDs. 
ISSN No:-2456-2165

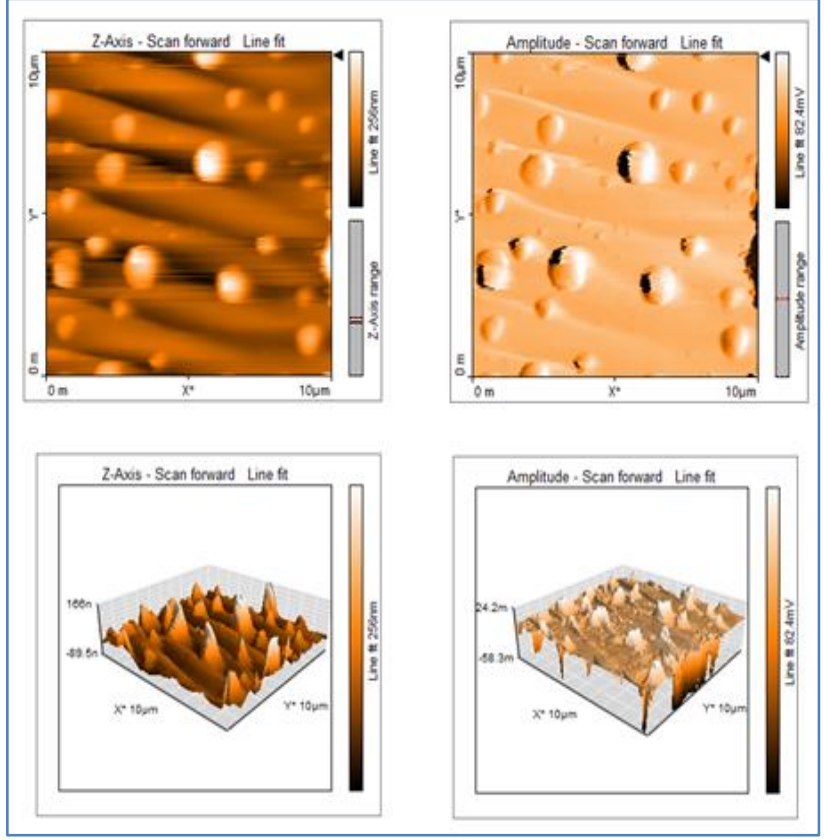

Fig 4:- AFM images of CDs (2D \& 3D image)

The image shows the particle size of CDs, which is in between $30 \mathrm{~nm}$ to $40 \mathrm{~nm}$. The particle size was calculated by the Nanosurf C3000 model software. The software can be easily detected the height of the particle, which was placed and dried at the top of the MICA plate. CDs have hydrophilic property, so some particles become clusters to joint with each other in atmosphere. The size can also be calculated from the $3 \mathrm{D}$ image of $\mathrm{CDs}$, which shows the surface of the MICA plate and some CDs particles are placed on it.

\section{Characterization study of CDs based Gel formulation}

As before, we discussed about the characterization study of CDs. Here is the characterisation study of CDs based Gel formulation. In figure.5 shows the UV-Vis spectra of the gel/ink. The graph shows the presence of the polymer, which was mixed with the carbon dots to form gel formulation. The graph shows some oscillator peaks between $650 \mathrm{~nm}$ to $750 \mathrm{~nm}$ wavelength.

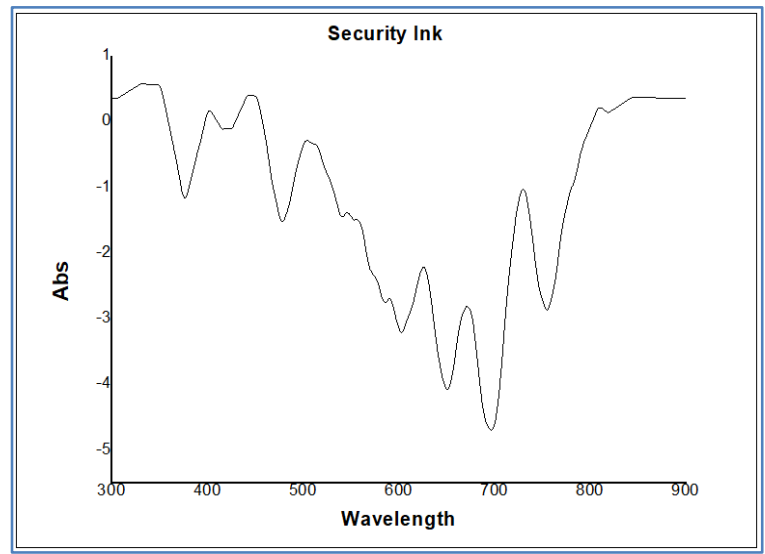

Fig 5:- UV-Vis spectra of Gel/ink
The graph also indicates little bit dissociation energy before $650 \mathrm{~nm}$ wavelength. It has maximum intensity at $700 \mathrm{~nm}$ wavelength. The peak is shift after mixing the carbon dot into polyurethane solution. The absorption peak shows between $350 \mathrm{~nm}$ to $450 \mathrm{~nm}$, which is shifted between $650 \mathrm{~nm}$ to $750 \mathrm{~nm}$. But still it has blue fluorescence absorbs at its highest intensity. But it is also depend on the parameters of the spectroscopy.

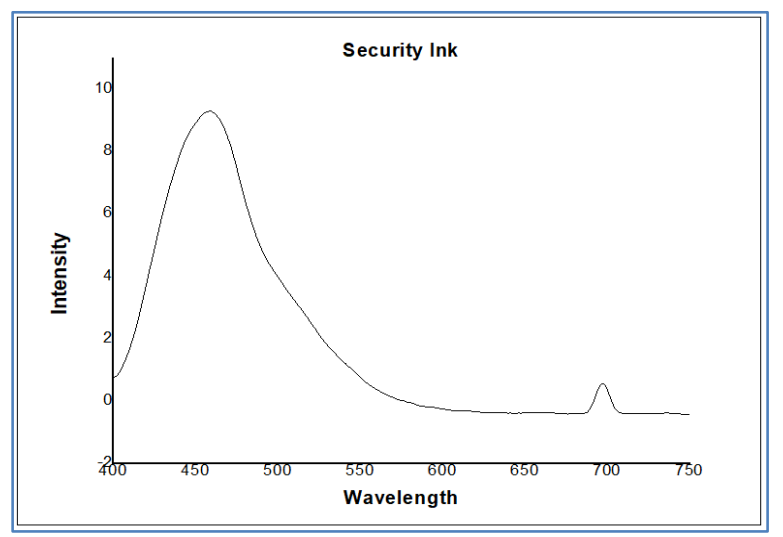

Fig 6:- Fluorescence spectra of Gel/ink

Figure.6 shows the fluorescence spectra of the gel, which concluded that after mixing the polymer with the carbon dots; it gives blue fluorescence colour at the highest and intense absorption peak at $400 \mathrm{~nm}$. The fluorescence absorption peak is shifted from $380 \mathrm{~nm}$ to $400 \mathrm{~nm}$ because of the presence of the polymer in the solution. The blue fluorescence colour is easily visible in the UV-Vis chamber. Figure.7 shows the comparison of fluorescence property of carbon dot and carbon dot based gel formulation.

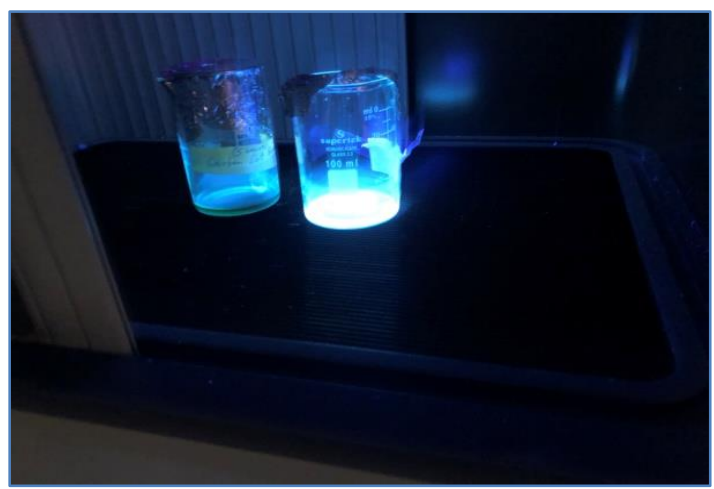

Fig 7:- Fluorescence comparison of carbon dots and carbon dot based gel formulation

In the image, the first beaker on the left hand side shows the blue fluorescence of carbon dot based gel formulation and on the right hand side beaker shows the fluorescence of carbon dots. The right hand side beaker clearly shows the intense blue colour fluorescence of CDs and the left hand side beaker has blue colour fluorescence, but the intensity is bit low because of the presence of the polymer in it. With changing the quantity of the carbon dot in polyurethane solution, the fluorescence spectra and intensity are also changed. 


\section{CONCLUSION}

By one step synthesising carbon dots, the hydrothermal method is very accurate and precise. It gives the crystalline and much fined nanoparticles of carbon. By one day process of synthesising, having blue fluorescence carbon dots are ready for further characterisation. It has $30 \mathrm{~nm}$ to $40 \mathrm{~nm}$ particle size with high intense luminescence peak at $380 \mathrm{~nm}$. With the help of the ethanolamine as a precursor, the carbon dot solution made transparent. So the transparent and fluorescence solution has many applications in bio-imaging, energy sector, security purpose (anti-counterfeiting), drug delivery, etc.

\section{REFERENCES}

[1]. B. Gayen, S. Palchoudhury, and J. Chowdhury, "Carbon dots: A mystic star in the world of nanoscience," J. Nanomater., vol. 2019, 2019.

[2]. X. Xu et al., "Electrophoretic analysis and purification of fluorescent single-walled carbon nanotube fragments," J. Am. Chem. Soc., vol. 126, no. 40, pp. 12736-12737, 2004.

[3]. Y. P. Sun et al., "Doped carbon nanoparticles as a new platform for highly photoluminescent dots," $J$. Phys. Chem. C, vol. 112, no. 47, pp. 18295-18298, 2008.

[4]. S. Liu et al., "Hydrothermal treatment of grass: A low-cost, green route to nitrogen-doped, carbon-rich, photoluminescent polymer nanodots as an effective fluorescent sensing platform for label-free detection of Cu(II) ions," Adv. Mater., vol. 24, no. 15, pp. 20372041, 2012.

[5]. M. d'Amora and S. Giordani, "Carbon Nanomaterials for Nanomedicine," Smart Nanoparticles Biomed., pp. 103-113, 2018.

[6]. Y. Wang and A. Hu, "Carbon quantum dots: Synthesis, properties and applications," J. Mater. Chem. C, vol. 2, no. 34, pp. 6921-6939, 2014.

[7]. R. Liu, D. Wu, S. Liu, K. Koynov, W. Knoll, and Q. $\mathrm{Li}$, "An aqueous route to multicolor photoluminescent carbon dots using silica spheres as carriers," Angew. Chemie - Int. Ed., vol. 48, no. 25, pp. 4598-4601, 2009.

[8]. Q. Tang, W. Zhu, B. He, and P. Yang, "Rapid Conversion from Carbohydrates to Large-Scale Carbon Quantum Dots for All-Weather Solar Cells," ACS Nano, vol. 11, no. 2, pp. 1540-1547, 2017.

[9]. X. K. Wan, Q. Tang, S. F. Yuan, D. E. Jiang, and Q. M. Wang, "Au19 nanocluster featuring a v-shaped alkynyl-gold motif," J. Am. Chem. Soc., vol. 137, no. 2, pp. 652-655, 2015. 